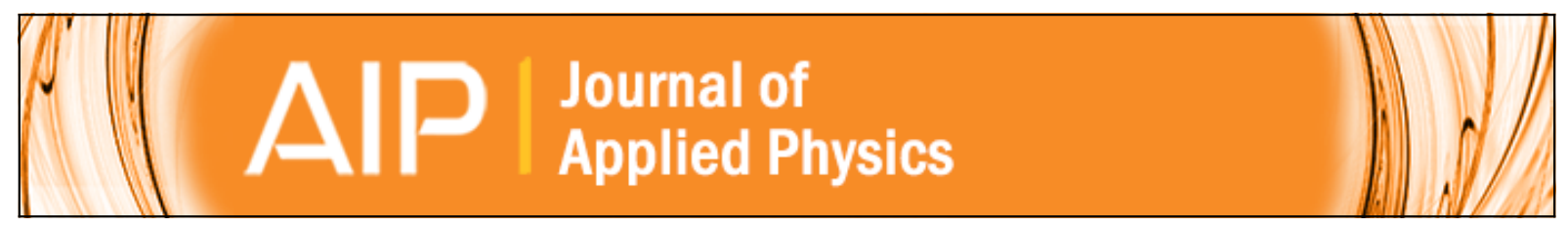

\title{
Numerical investigation of flow-through immunoassay in a microchannel
}

A Sinha, R Ganguly, and I K. Puri

Citation: Journal of Applied Physics 107, 034907 (2010); doi: 10.1063/1.3284077

View online: http://dx.doi.org/10.1063/1.3284077

View Table of Contents: http://scitation.aip.org/content/aip/journal/jap/107/3?ver=pdfcov

Published by the AIP Publishing

\section{Articles you may be interested in}

Translocation of nanoparticles through a polymer brush-modified nanochannel

Biomicrofluidics 6, 034101 (2012); 10.1063/1.4732799

Wafer-scale fabrication of high-aspect ratio nanochannels based on edge-lithography technique

Biomicrofluidics 6, 016502 (2012); 10.1063/1.3683164

Isolation and manipulation of living adherent cells by micromolded magnetic rafts

Biomicrofluidics 5, 032002 (2011); 10.1063/1.3608133

Optofluidic Fabry-Pérot cavity biosensor with integrated flow-through micro-/nanochannels Appl. Phys. Lett. 98, 041104 (2011); 10.1063/1.3548673

Compact electromagnetically operated microfluidic system for detection of sub-200-nm magnetic labels for biosensing without external pumps

J. Appl. Phys. 107, 09B313 (2010); 10.1063/1.3352584

MIT LINCOLN

LABORATORY

CAREERS

Discover the satisfaction of innovation and service

to the nation
- Space Control

- Air \& Missile Defense

- Communications Systems \& Cyber Security

- Intelligence, Surveillance and

Reconnaissance Systems
- Advanced

Electronics

- Tactical Systems

- Homeland

Protection

- Air Traffic Control

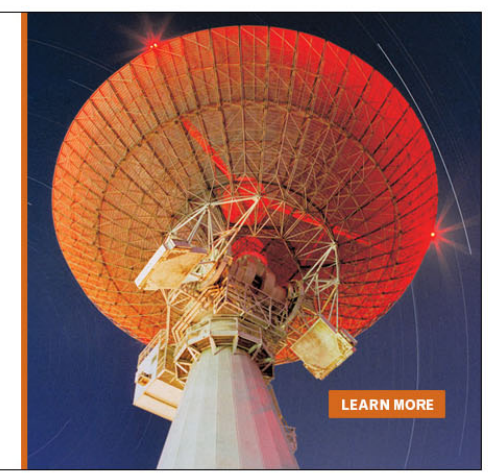




\title{
Numerical investigation of flow-through immunoassay in a microchannel
}

\author{
A Sinha, ${ }^{1}$ R Ganguly, ${ }^{2}$ and I K. Puri, ${ }^{1}$ a) \\ ${ }^{1}$ Department of Engineering Science and Mechanics, Virginia Polytechnic Institute and State University, \\ Blacksburg, Virginia 24061, USA \\ ${ }^{2}$ Department of Power Engineering, Jadavpur University, Kolkata 700098, India
}

(Received 20 June 2009; accepted 7 December 2009; published online 9 February 2010)

\begin{abstract}
Immunomagnetic separation (IMS) is a method to isolate biomaterials from a host fluid in which specifically selected antibodies attached to magnetic particles bind with their corresponding antigens on the surface of the target biological entities. A magnet separates these entities from the fluid through magnetophoresis. The method has promising applications in microscale biosensors. We develop a comprehensive model to characterize the interaction between target species and magnetic particles in microfluidic channels. The mechanics of the separation of target nonmagnetic $N$ particles by magnetic $M$ particles are investigated using a particle dynamics simulation. We consider both interparticle magnetic interactions and the binding of the functionalizing strands of complementary particles. The temporal growth of a particle aggregate and the relative concentrations of $M$ and $N$ particles are investigated under different operating conditions. A particle aggregate first grows and then exhibits periodic washaway about a quasisteady mean size. The washaway frequency and amplitude depend on the initial fractional concentration of $N$ particles while the aggregate size scales linearly with the dipole strength and inversely with the fluid flow rate. () 2010 American Institute of Physics. [doi:10.1063/1.3284077]
\end{abstract}

\section{INTRODUCTION}

Advances in immunology have resulted in a variety of diagnostic techniques and cellular probes, ${ }^{1}$ e.g., for immunomagnetic separation (IMS), ${ }^{2,3}$ which use magnetic particles with surface immobilized antibodies that specifically bind with various antigens on the surface of target cells, DNA strands or other biological entities to form conjugates. ${ }^{4}$ After binding, a high gradient magnetic field is employed to retain the immunochemically bound entities in a container while washing away magnetically unresponsive nontarget cells or molecules. ${ }^{3}$ Sequential activation and deactivation of the external field enables cyclic collection and resuspension of the particles for successive wash steps. The remnant is a concentrated and purified suspension of the target species labeled with magnetic particles. The primary purpose of magnetic separation is to concentrate the sample to attain a higher signal to noise ratio of detection than is possible with an untreated suspension, thus eliminating the need for sample pretreatment and amplification of the target concentration. Magnetic separation can therefore be advantageous over physical concentration methods such as filtration and centrifugation, which can lead to loss of sample integrity.

The IMS technique can be implemented in either a batch mode or a continuous mode separation. Batch mode separators are limited to relatively small sample volumes, ${ }^{5}$ an open operating environment, and relatively long reaction times that are constrained by the particle and analyte concentrations. These characteristics preclude their use for many physiological and environmental sensor applications for which sample enrichment is undesirable or impossible and

\footnotetext{
${ }^{a)}$ Author to whom correspondence should be addressed. Electronic mail: ikpuri@vt.edu. Tel.: +1-540-231-3243. FAX: +1-540-231-4574.
}

continuous (flow through) separation is preferred over batch mode separation from an automation perspective. During a flow-through magnetic separation process, the channel is continuously flushed with the sample and functionalized particles. Strategically placed magnets on the channel walls result in the cross-stream migration of the particles, thereby enhancing their interactions with the target species. This interaction binds the magnetic particles with the target species. The particle-target aggregate is thereafter magnetically immobilized on the channel wall closest to the magnet [Fig. 1(a)]. Finally, the background fluid is eluted by a buffer in a washing step, leaving behind the magnetically immobilized particle-target conjugate. ${ }^{6}$ In contrast to its batch counterpart,
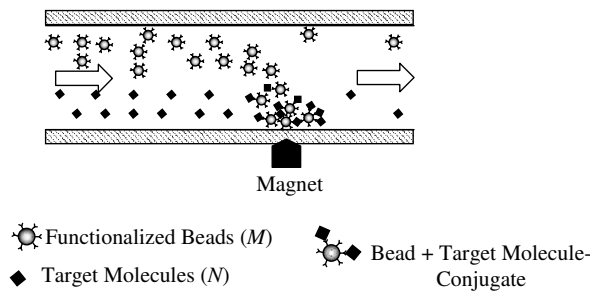

(a)

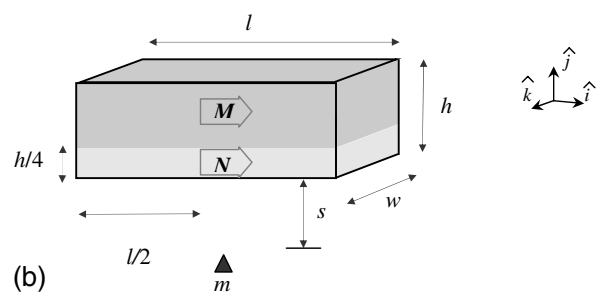

FIG. 1. (a) The concept of IMS. (b) Schematic of simulation domain. The simulation conditions are $a=500 \mathrm{~nm}, \chi=0.1, U o=$ variable, $s=15 \mu \mathrm{m}, \varphi$ $=0.7,\left.\quad n_{M}\right|_{t=0}+\left.n_{N}\right|_{t=0}=30, \quad h=w=20 \mu \mathrm{m}, \quad R_{c}=1.01, \quad R_{a}=1.01, \quad$ and $m$ $=$ variable. 
the flow-through method has a potential for high purity and recovery, multiplexing, and continuous operation, thus enabling continuous sensing.

The primary challenges to successful implementation of the IMS technique in flow-through immunoassay devices is to ensure (1) a reasonable extent of immunochemical binding of the target entities on the beads in situ and (2) a selective separation of the bead-target conjugates from the background fluid. Practical microfluidic IMS devices are expected to operate under a wide range of concentrations of the target analyte, sample loadings ranging from very dilute (e.g., in case of an online biosensor for pathogen detection) to very dense (e.g., for a cell sorter). It is therefore important to ascertain the correct loading of the magnetic beads in the separator for a particular task. While a large magnetic bead density is wasteful, fewer beads can lead to inadequate separation. In a biosensor application, the separated sample of a bead-target conjugate can be used for the purpose of detection. Therefore it is also important to know the composition of the separated sample in a flow-through microfluidic IMS device.

Magnetic particles have been used in microchannels ${ }^{7}$ and are suitable in the implementation of massively parallel IMS microfluidic networks to enable more robust process automation. ${ }^{8}$ The initial feasibility studies ${ }^{9-11}$ in this regard have been mostly empirical ${ }^{12}$ and there are few comprehensive models available that describe the interaction between target species and magnetic particles. ${ }^{5}$ We therefore conduct a fundamental investigation of the mechanics of magnetic separation on a microfluidic platform using threedimensional numerical simulations. ${ }^{3,13}$ Previous studies have assumed binding when the gap between a particle and the target is smaller than a critical distance. ${ }^{5}$ A mobility- and diffusion-dominated binding kinetics model can also be employed where every collision is assumed to result in particletarget binding. ${ }^{14}$ To separate particles larger than $1 \mu \mathrm{m}$ (that are characteristic of cell separation applications) using magnetic beads of similar size, immunomagnetic binding is more strongly influenced by the particle mobility and the collision rates. In a dense suspension, and in the regions of high magnetic field gradients of the immunomagnetic separators, particle collision is strongly influenced by short-range interparticle dipolar interaction.

We extend magnetic particle dynamic simulations ${ }^{15}$ to the immunomagnetic binding and dynamics of bead-target cell conjugates in microfluidic configurations. The covalent bonds that represent biochemical functionalization, which cannot be broken by fluid shear forces or by particle collisions, are represented herein though a more elegant velocity interaction model. We use the model to investigate the influence of changing relative concentrations of target cells and magnetic beads on the aggregation dynamics. Influence of the dipole strength and flow velocity on the aggregate size is also investigated.

\section{MODEL}

A schematic of the simulation domain is presented in Fig. 1(b). The channel has a length $l$ and a square cross section defined by $w=h$. The magnetic particles $M$ and the nonmagnetic target cells $N$ in our simulation are identical in their physical properties, except for the absence of magnetic properties in the latter. The two fluids carrying $M$ and $N$ particles enter through the top $(3 h / 4)$ and bottom $(h / 4)$ left sections of the channel, respectively. A point dipole, ${ }^{3}$ situated at a distance $s$ below the geometric center of the lower channel boundary and $l / 2$ downstream of its inlet, is used to produce the requisite magnetic field. The dipole is oriented with its axis aligned with the $y$-axis. The suspension is assumed to contain monodisperse, neutrally buoyant, and frictionless micron sized spherical magnetic particles of radius $a$ and density $\rho_{p}$. The particles are assumed to be nonBrownian, since motion of the $1 \mu \mathrm{m}$ particles due to thermal fluctuations is negligible as compared to movements induced by the flow and the range of magnetophoretic velocities. ${ }^{16}$ We assume that the fluid drag on the particles influences the particle trajectories, but a reciprocal reaction of the particles does not alter the fluid flow. This is a reasonable assumption for a fluid with a small particle loading or concentration, which is the case here. Therefore, the velocity distribution in the fully developed pressure-driven flow region of a microchannel follows the infinite Fourier series solution of the Stokes equation ${ }^{17}$

$$
\begin{aligned}
\mathbf{u}^{f}(y, z) & =\left(\frac{G h^{2}}{8 \eta}\right) \frac{32}{\pi^{3}} \sum_{n=0}^{\infty}\left[\left(\frac{(-1)^{n}}{(2 n+1)^{3}}\right)\right. \\
& \times\left\{1-\frac{\cosh \left\{\frac{(2 n+1) \pi y}{h}\right\}}{\cosh \left\{\frac{(2 n+1) \pi w}{2 h}\right\}}\right\} \\
& \times \cos \left\{\frac{(2 n+1) \pi z}{h}\right\} \hat{i} .
\end{aligned}
$$

The peak centerline velocity $\left(U_{0}\right)$ in the channel is varied by adjusting the pressure gradient $G=-d p / d x$.

The Newtonian relation for particle motion is $(4 / 3) \pi a^{3} \rho_{p} \dot{\mathbf{u}}_{i}=\mathbf{F}_{i}^{m}+\mathbf{F}_{i}^{d}$, where $\mathbf{F}_{i}^{m}$ and $\mathbf{F}_{i}^{d}=6 \pi \eta a\left(\mathbf{u}_{i}-\mathbf{u}^{f}\right)$ denote the magnetic force and the viscous drag, respectively, where $\eta$ denotes the dynamic viscosity of the fluid. The induced magnetic moment within each composite magnetic microparticle $\mathbf{m}_{i}=\chi \mathbf{H}_{i} V \varphi$ depends upon the saturation magnetization of the $5 \mathrm{~nm}$ particles that are assumed to be embedded within it, the volume fraction $\varphi$ of the magnetic content due to these nanoparticles within the microspheres, and the particle volume $V$. The very small particle Reynolds number $\left(\sim 10^{-3}\right)$ for this flow implies that the effect of particle inertia is negligible as compared to viscous effects. ${ }^{18}$ Hence, $\mathbf{F}_{i}^{m}=-\mathbf{F}_{i}^{d}$ so that the magnetophoretic velocity $\mathbf{u}_{i}$ $=(1 / 6 \pi \eta a) \mathbf{F}_{i}^{m}+\mathbf{u}^{f}$. The magnetic force $\mathbf{F}_{i}^{m}$ is related to the 
local field experienced by the $i$ th particle through $\mathbf{F}_{i}^{m}$ $=\mu_{0} V \varphi \chi_{\text {eff }} \frac{1}{2} \boldsymbol{\nabla}\left(\mathbf{H}_{i} \cdot \mathbf{H}_{i}\right)$ while the effective magnetic susceptibility $\chi_{\text {eff }}$ of each spherical particle is described in terms of its intrinsic susceptibility through the relationship $\chi_{\text {eff }}$ $=\chi^{\prime}\left(1+\frac{1}{3} \chi\right),{ }^{18}$ which accounts for the self demagnetization due to the field produced by the $i$ th particle.

In a concentrated suspension, interactions between magnetized particles result in an attractive force between them. A particle distorts the field in its vicinity so that the force on a neighboring particle also changes, which provides the basis for the interaction between adjacent point dipoles. The local field on the $i$ th particle due to the neighboring $j$ th particles $\mathbf{H}_{i}=\mathbf{H}_{0}+\sum_{j=1, i \neq j}^{N}(1 / 4 \pi)\left[3\left(\mathbf{m}_{j} \cdot \mathbf{r}_{i j}\right) \mathbf{r}_{i j}-\mathbf{m}_{j} r_{i j}^{2}\right] / r_{i j}^{5}$, where $\mathbf{H}_{0}$ is the applied field due to the external dipole $\mathbf{m}=m \hat{j}$. Close lying $M-M$ particle pairs are attracted to each other through magnetic attraction, while the attraction between close lying $M-N$ particle pairs occurs due to immunochemical attraction. This latter interaction occurs due to the complementary functionalizing molecules present on their surfaces that are effective only at very close ranges. Both the magnetic and nonmagnetic interactions depend on the individual particle sizes and the interparticle separation.

When particles collide, unless a "stand-off distance" between the colliding particle centers is accounted for, the simulations can predict an unphysical overlapping of particles. In our model, particle overlap is prevented through a velocity-based collision barrier, ${ }^{19}$

$$
\mathbf{v}_{i j}^{r}=\frac{-V_{\mathrm{ref}}}{2 a}\left(\frac{R_{\mathrm{ref}}^{2}-r_{i j}^{2}}{R_{\mathrm{ref}}^{2}-4 a^{2}}\right)^{2} \mathbf{r}_{i j}, \quad \text { for } r_{i j}<R_{\mathrm{ref}},
$$

where $V_{\text {ref }}$ denotes the maximum overlap velocity among all the particle pairs in the microchannel domain at a particular instant of time, and $R_{\text {ref }}$ a cutoff distance below which $\mathbf{v}_{i j}^{r}$ $\neq 0$. Using normalized velocity and distance parameters, e.g., $\bar{V}=\left|\mathbf{v}_{i j}^{r}\right| / V_{\text {ref }}, \bar{R}=R_{\text {ref }} / 2 a$ (=1.01 for our simulations), and $\bar{r}=r_{i j} / 2 a$, Eq. (2) becomes

$$
\bar{V}=\left(\frac{\bar{R}^{2}-\bar{r}^{2}}{\bar{R}^{2}-1}\right)^{2} \bar{r} ; \quad \bar{r}:(1, \infty) .
$$

The behavior of this collision barrier is described through Fig. 2(a). Particles continue to approach one another under mutual attraction until they are separated by a distance $R_{\text {ref }}$. Once, $\bar{r}<\bar{R}$ (or $r_{i j}<R_{\text {ref }}$ ), $\bar{V}$ increases with decreasing $\bar{r}$. The selection of $\bar{R}$ determines whether the colliding particles behave as hard or soft spheres. Since, $R_{\text {ref }}$ can be arbitrarily selected under the constraint that any further reduction does not significantly alter the predicted particle dynamics, we use $\bar{R}=1.02$. At $\bar{r}=1$ and $\bar{V}=1$ for all $\bar{R}$, which corresponds to a 'kissing' configuration. Some particle pairs never attain this state because all particles are assigned a value of $V_{\text {ref }}$ based on the fastest approaching particle pair in the microchannel domain.

The viscoleastic nature of the functionalizing strands on the surfaces of the $M$ and $N$ particles is difficult to characterize. ${ }^{20}$ Thus, rather than resorting to the details of the binding forces we propose a velocity effect, which also produces unconditional binding similar to that observed in co-
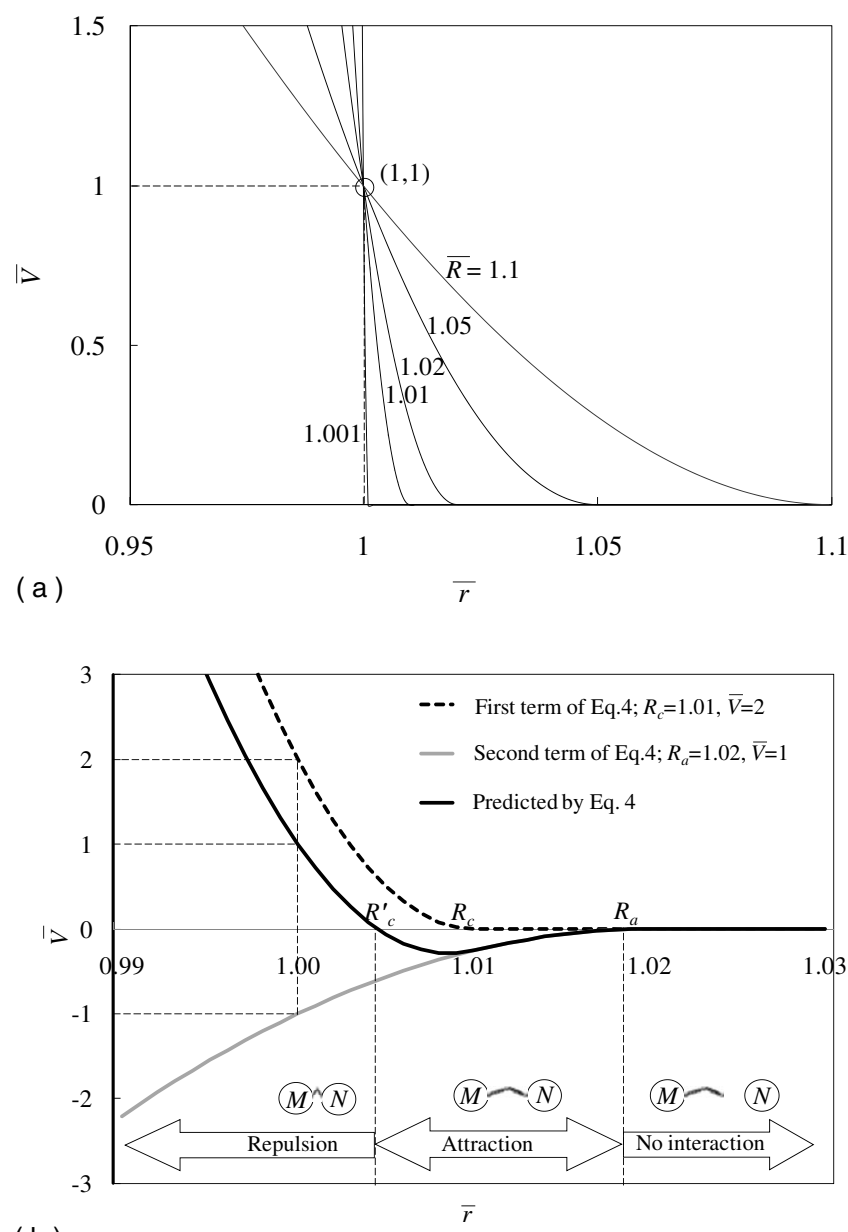

(b)

FIG. 2. (a) Increase in $\bar{V}$ for $\bar{r}<\bar{R}$ as predicted by Eq. (3). As $\bar{R} \rightarrow 1$, the collision model shifts from a soft to a hard sphere model. (b) The resulting velocity distribution model as predicted by Eq. (4), showing the three interaction regimes, of repulsion, attraction, and no interaction, respectively.

valent bonds. The velocity barrier collision model of Eq. (3) is thus modified by including an attraction term between an $M-N$ particle pair so that

$$
\begin{aligned}
& \bar{V}=2\left(\frac{\bar{R}_{c}^{2}-\bar{r}^{2}}{\bar{R}_{c}^{2}-1}\right)^{2} \bar{r}-\left(\frac{\bar{R}_{a}^{2}-\bar{r}^{2}}{\bar{R}_{a}^{2}-1}\right)^{2} \bar{r}, \\
& R_{c}=1.01, \quad R_{a}=1.02 .
\end{aligned}
$$

Henceforth, the $\bar{R}$ conditions related to collision and attraction will be, respectively, referred to as $R_{c}$ and $R_{a}$. Since reducing $\bar{R}$ below 1.02 does not change the system dynamics, we set $R_{a}=1.01$ and $R_{c}=\bar{R}$. The resulting velocity barrier described in Fig. 2(b) can be described through three regimes: pure repulsion (when $r_{i j}<R_{c}^{\prime}$ ), attraction (when $R_{c}^{\prime}$ $>r_{i j}>R_{a}$ ), and a third when there is no interaction between the particles. A conservative value for $V_{\text {ref }}$ is determined by examining the maximum hydrodynamic approach velocity between interacting particle pairs when $r_{i j}>R_{a}$. This reduces the effective value of $R_{c}$ for the model from the stipulated 1.01 to 1.005 , as shown in Fig. 2(b), which is referred to as $R_{c}^{\prime}$. 


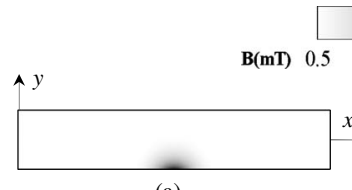

(a)

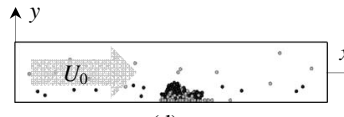

(d)

$\begin{array}{lllll}20.5 & 40.5 & 60.5 & 80.5 & 100.5\end{array}$

A $y \quad \wedge^{-} z$

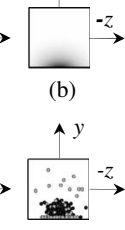

(e)
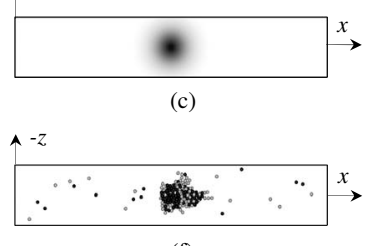

(f)

FIG. 3. (a) Side view ( $z=0$ plane), (b) End view ( $x=l / 2$ plane) and (c) Bottom view $(y=-h / 2$ plane) of the profile of the magnetic field created by a point dipole. The grayscale bar represents the magnetic field strength in millitesla (mT). (d) Side view, (e) end view, and (f) bottom view of images of the simulated particle aggregation at steady state. The filled and empty circles represent $N$ and $M$ particles, respectively. For this simulation, $c_{0}$ $=0.6$.

The instantaneous particle positions are obtained through time integration of the particle velocities,

$$
\mathbf{x}(t)=\int_{0}^{t} \mathbf{u}_{p} d t+\mathbf{x}_{0}
$$

using the Euler explicit integration method. The forward differencing time step for the simulation is primarily constrained by a travel distance per time step that is less than the width of the attraction/repulsion zone, or 1/20th of the particle size, whichever is less, i.e., $\left|\mathbf{u}_{p}\right| \times \delta t<\min \left[\left\{R_{\text {ref }}\left(R_{a}\right.\right.\right.$ $\left.\left.\left.-R_{c}^{\prime}\right) / 10\right\} ;(a / 20)\right]$.

\section{RESULTS AND DISCUSSION}

Simulations are performed in an $h=w=20 \mu \mathrm{m}$ and $l$ $=100 \mu \mathrm{m}$ microchannel for $a=500 \mathrm{~nm}$ particles with $\chi$ $=0.1$ and $\varphi=0.7$. Parametric investigations are conducted for different values of $U_{0}, m$, and the fractional number densities of nonmagnetic particles. For a steady flow and particle influx rate, the particle entry and exit rates for the microchannel domain are constant, as are the numbers of $M$ and $N$ particles within it. Based on a given inlet particle flux, the average number of particles in the domain before the dipole is activated as

$$
\left.n_{M}\right|_{t=0}+\left.n_{N}\right|_{t=0}=30,
$$

which, for the domain size considered corresponds to an absolute particle number density of $0.75 \times 10^{15} / \mathrm{m}^{3}$. The intial fractional concentration of nonmagnetic particles, denoted by $c_{0}=\left.c(t)\right|_{t=0}=\left.\left[n_{N} /\left(n_{N}+n_{M}\right)\right]\right|_{t=0}$, is varied by changing $\left.n_{N}\right|_{t=0}$ and $\left.n_{M}\right|_{t=0}$ with Eq. (6) as a constraint. Once the dipole is activated, $M$ and $N$ particles are captured, increasing the particle count $n_{M}+n_{N}$ beyond the initial number of 30. Depending upon the number of $M$ and $N$ particles captured, $c(t)$ also changes.

Figures 3(a)-3(c) show three views of the magnetic field contours produced by the point dipole for a representative case of $m=9 \times 10^{-12} \mathrm{~A} \mathrm{~m}^{2}$. The maximum value of $|\mathbf{B}|$ $=100 \mathrm{mT}$ occurs closest to the dipole but rapidly decreases away from the dipole. Figures 3(d)-3(f) present an instantaneous simulation snapshot for a steady flow with $U_{0}$ $=10 \mu \mathrm{m} / \mathrm{s}$ and $c_{0}=0.6$ at time $t=850 \mathrm{~s}$ after the magnetic field is activated. The particles migrate and collect in the

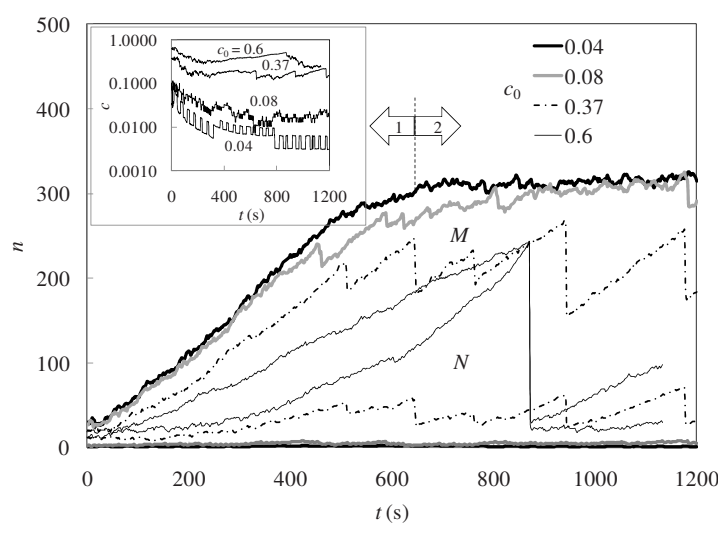

FIG. 4. Aggregate size vs time. The curves passing through the shaded area labeled as $M$ refer to $n_{M}$, while those passing through the corresponding $N$ area represent $n_{N}$. The inset shows the behavior of $c(t)$.

high field gradient region near the point dipole as shown in Figs. 3(d)-3(f). Particles lying on the periphery of the aggregate describe a boundary along which the aggregate-holding force due to the magnetic field competes with the aggregatedepleting drag force. As the aggregate size increases, its outer boundary moves progressively further away from the wall. Consequently, the values of the magnetic field strength and its gradient at this boundary, both of which attract particles from the flow into the aggregate, diminish over time. Eventually, the aggregate-holding magnetic force at the boundary becomes comparable to the depleting flow-induced drag force so that, despite particle addition into the domain, aggregate growth ceases. On average, the numbers of particles added to the aggregate and removed from it are equal at this stage although, due to the granular nature of the aggregate, changes in its size due to shearing, while never significantly large are nevertheless discrete.

Figure 4 presents the temporal evolution of the numbers of $M$ and $N$ particles retained in the domain for various $c_{0}$ when the initial $\left(n_{M}+n_{N}\right)$ particle count starts from 30. After the dipole is activated at $t=0$, magnetic particles are progressively collected on the channel wall. When $c_{0}=0.04$, the flow contains mostly $M$ particles. This collection phase lasts $\approx 500 \mathrm{~s}$ until the aggregate reaches a critical size that marks the stage between the aggregate buildup and washaway phases. In this case, the buildup phase occurs in the region labeled Zone 1 in Fig. 4 and its duration depends on various system variables (fluid velocity, particle magnetization, and size, etc.). During the washaway phase (marked Zone 2 in Fig. 4), average number of particles lost roughly equals those collected, resulting in an approximately constant total particle number for an aggregate. There are temporal fluctuations in the $n_{M}$ and $n_{N}$ versus $t$ plots during the washaway phase that is characterized by sudden losses of the particles from the aggregate followed by rapid accumulation. Denoting the time-averaged values during the washaway phase by the superscript ${ }^{*}, n^{*}=\int_{\tau} n d t / \tau$, where $\tau$ denotes a complete washaway cycle. The aggregate size for $c_{0}=0.04$ corresponds to $n_{M}^{*}=320$ and $n_{N}^{*} \approx 2$. The aggregate buildup rate is linear for $M$ particles but quadratic for $N$ particles. The enlarging aggregate forms a growing obstacle within the flow as more $M$ particles are collected, allowing a larger surface area to 
become exposed to the flow for the immobilization of $N$ particles on it. Due to the relatively small number of $N$ particles for small $c_{0}$ values, the presence of $N$ particles on the aggregate is mostly limited to the outer surface. Figure 4 also provides insight into the size evolution of an aggregate as $c_{0}$ is varied. Here, $n_{\max }\left(=n_{N}+n_{M}\right)$ is indicative of the critical size of the aggregate before the onset of washaway. The $n_{M}$ and $n_{N}$ curves in Fig. 4 show similar trends for all $c_{0}$ values although $n_{M}$ and $n_{N}$ differ. Larger $c_{0}$ values produce smaller $n_{M}$ and larger $n_{N}$ values.

Particle shedding from the aggregate occurs differently in Zones 1 and 2. In Zone 1, washaway occurs through the shearing of individual particles, but in Zone 2 larger chunks of the aggregate are also washed away. The shedding of particles in Zone 2 is strongly dependent on $c_{0}$. As $c_{0}$ increases, the shedding amplitude (i.e., the fraction of the aggregate shed in a cycle) increases, while the shedding frequency decreases. For $c_{0}=0.6$, almost $90 \%$ of the aggregate is washed away during a single cycle. In Zone 2, interparticle forces within the aggregate that lead to bulk interaction forces are as important as the fluid shear force. From the perspective of practical device design, the simulation refers to a separation section that concentrates on the interacting $M$ and $N$ particles into an aggregate. Some bound $M-N$ conjugates are released and convected downstream from this aggregate through washaway.

The inset of Fig. 4 shows that $c(t)$ initially decreases as more $M$ particles than $N$ particles are captured by the aggregate. Subsequently, as the aggregate surface grows, $N$ particle capture also increases and $\left.c(t)\right|_{t \rightarrow \infty}$ reaches a quasisteady state. For larger values of $c_{0}$, the rate of $M$ particle addition to the aggregate during the buildup phase is smaller, while the corresponding values of $d\left(n_{N}\right) / d t$ are larger. Therefore, with increasing $c_{0},\left.c(t)\right|_{t \rightarrow \infty}$ is also larger due to the higher fraction of $N$ particles being captured. For $c_{0}=0.04$, the time averaged particle count $\left.c(t)\right|_{t \rightarrow \infty}=0.006$ (or 15\% of the corresponding $c_{0}$ value), but at $c_{0}=0.37\left[\left.c(t)\right|_{t \rightarrow \infty}=0.18\right.$, which is $47 \%$ of the corresponding $c_{0}$ ].

The aggregate volume is related to the volume fractions $P_{M}^{*}=n_{M}^{*} V_{P} / V$ and $P_{N}^{*}=n_{N}^{*} V_{P} / V$, where $V_{P}=4 \pi a^{3} / 3$ denotes the volume of a particle and $V=l w h$ the microchannel (or domain) volume. Figure 5(a) presents the time averaged values of $P_{M}^{*}$ and $P_{N}^{*}$ during the washaway phase and Fig. 5(b) presents their standard deviations $\left(\sigma_{M}\right.$ $=\sqrt{\sum_{k}\left(n_{M}-n_{M}^{*}\right)^{2} /(k-1)}$ and $\sigma_{N}=\sqrt{\left.\sum_{k}\left(n_{N}-n_{N}^{*}\right)^{2} /(k-1)\right)}$ as a function of $c_{0}$. Larger $c_{0}$ values correspond to relatively larger numbers of $N$ particles for which $M-N$ interactions are more probable but $M-M$ interactions less so, washaway occurs more readily for larger $c_{0}$ as the numbers of $M$ particles become insufficient to form tightly bound aggregates so that both $P_{N}^{*}$ and $P_{M}^{*}$ approach zero as $c_{0} \rightarrow 1$. Conversely, as $c_{0}$ $\rightarrow 0$, negligibly small amounts of $N$ particles are collected.

As $c_{0}$ increases in the range $0-0.75$, the values of $P_{M}^{*}$ decrease, while those of $\sigma_{M}$ and $P_{N}^{*}$ increase. At $c_{0} \approx 0.75$, $P_{M}^{*} \approx P_{N}^{*}$, indicating that an equal number of $M$ and $N$ particles are immobilized in the aggregate and its washed away portion. If $c_{0}$ increases further, both $P_{M}^{*}$ and $P_{N}^{*}$ decrease. Between $c_{0}=0.75-1$, the relative concentrations of both $M$ and $N$ particles immobilized in the aggregate are roughly
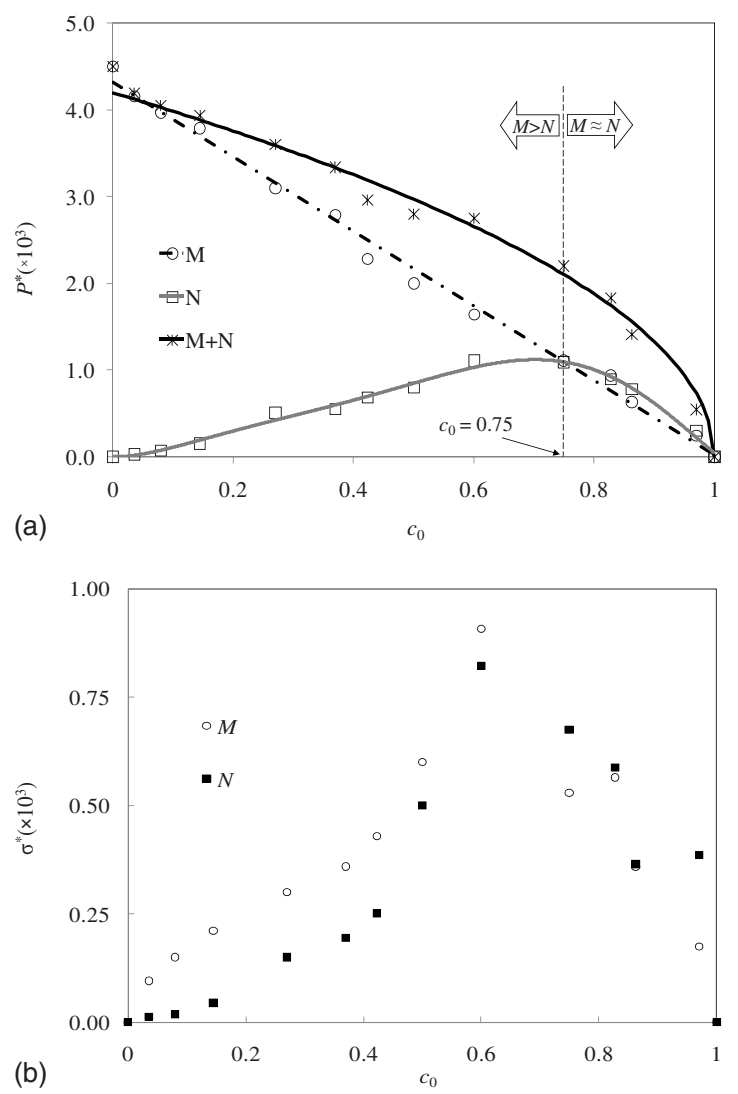

FIG. 5. (a) The critical aggregate size during the washaway phase vs the relative concentration of $M$ and $N$ particles. (b) The standard deviations $\sigma_{M}$ and $\sigma_{N}$ of particle count in the aggregate during the washaway phase.

equal although the number of $M-N$ conjugates always decreases with increasing $c_{0}$. When $c_{0}=1$, the system contains no $M$ particles to initiate aggregation so that there is zero collection. Therefore, for a specified inlet particle loading, $c_{0}=0.75$ is an optimal operating condition when the largest number of $N$ particles are immobilized in the aggregate. Figure 5(b) presents the change in $\sigma_{M}$ and $\sigma_{N}$ with respect to varying $c_{0}$. Close to $c_{0}=0.6$, the aggregate experiences complete cyclic washaway, i.e., it is entirely lost periodically, with the consequence that $c(t) \rightarrow c_{0}$. This standard deviation increases with increasing $c_{0}$ for both $M$ and $N$ particles and reaches a maximum value at $c_{0}=0.6$. All simulations for $c_{0}$ $>0.6$ produce washaway. The $\sigma_{M}$ and $\sigma_{N}$ values decrease and approach zero as $c_{0} \rightarrow 1$.

Figure 6 illustrates the influence of $m$ and $U_{0}$ on $P_{M}^{*}$ and $P_{N}^{*}$ for $c_{0}=0.36$. When $m$ increases, so does $\mathbf{H}$ and its gradient, which produces a corresponding increase in the collection of both $M$ and $N$ particles. Figure 6(a) shows a linear variation of $P^{*}$ with $m$ for $U_{0}=10 \mu \mathrm{m} / \mathrm{s}$, similar to previous simulations with only $M$ particles. ${ }^{21}$ The $P^{*}$ plots of Fig. 6(a) correspond to $\left.c(t)\right|_{t \rightarrow \infty} \approx 0.19$, indicating that the dipole strength has little effect on the $N-M$ composition of the final aggregate. As shown in Fig. 6(b), $P^{*} \propto U_{0}^{-1}$, since increasing $U_{0}$ also increases the shear at the aggregate periphery, thereby producing smaller aggregates. Increasing $U_{0}$ $>30 \mu \mathrm{m} / \mathrm{s}$ results in negligible collection of both $M$ (hence $N)$ particles. In contrast to a pure aggregate of $M$ particles for which the aggregate size scales with $U_{0}^{-2 / 3},{ }^{21}$ the fluid velocity has a stronger depleting influence on $M-N$ aggregates. 

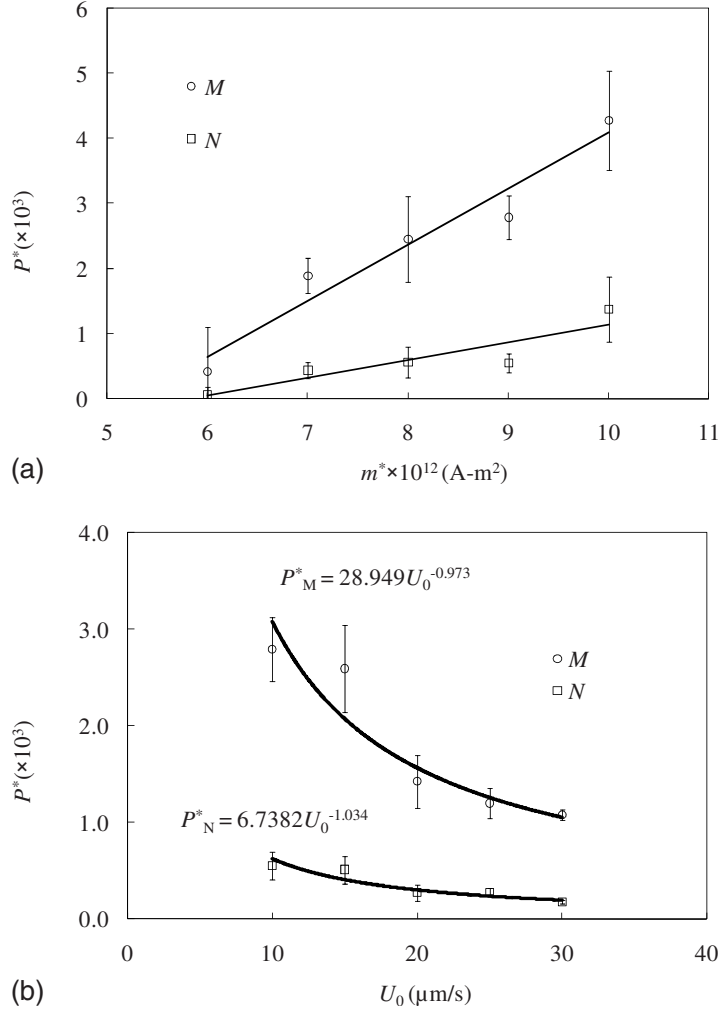

FIG. 6. Variation in $P_{M}^{*}$ and $P_{N}^{*}$ as a function of (a) dipole strength $m$ (at $U o=10 \mu \mathrm{m} / \mathrm{s}$ ), and (b) peak centerline velocity $U_{0}$ (at $m=9$ $\left.\times 10^{-12} \mathrm{~A}-\mathrm{m}^{2}\right)$. Here, $c_{0}=0.36$ in both cases (a) and (b).

\section{CONCLUSIONS}

We simulate the immunomagnetic binding of a target species $N$ with magnetic particles $M$ of identical size and their subsequent separation in a microfluidic environment. A modified velocity barrier collision model includes a shortrange attraction term between $M-N$ particles, providing the requisite binding, while a magnetic dipolar interaction model describes the attraction force between $M-M$ particles. A point dipole is used for magnetophoretic separation of the $M$ particles and the $N$ particles to which they are bound. A particle aggregate forms close to the dipole and grows to a quasisteady size although periodic bulk washaway produces fluctuations in this size about a mean value. The larger the number of $N$ particles that are collected, the greater is this temporal fluctuation until $c_{0}=0.6$. For a specified total particle loading, the fraction of $N$ particles in the aggregate increases with $c_{0}$. However, the optimal operating condition corresponds to $c_{0}=0.75$ when the largest number of $N$ particles are immobilized in the aggregate. The composite $M-N$ aggregate behaves differently than a pure aggregate of $M$ particles. Varying the magnetic strength and fluid velocity shows that $P^{*} \propto\left(m, U^{-1}\right)$ for specified relative concentrations of $M$ and $N$ particles. The analysis provides a rational basis to select the operating conditions for a microfluidic IMS device and predict its performance.

${ }^{1}$ I. Safarik and M. Safariková, J. Chromatogr., B: Biomed. Sci. Appl. 722, 33 (1999).

${ }^{2}$ O. Olsvik, T. Popovic, E. Skjerve, K. S. Cudjoe, E. Hornes, J. Ugelstad, and M. Uhlen, Clin. Microbiol. Rev. 7, 43 (1994).

${ }^{3}$ A. Sinha, R. Ganguly, A. K. De, and I. K. Puri, Phys. Fluids 19, 117102 (2007).

${ }^{4}$ H. Yu, J. W. Raymonda, T. M. McMahon, and A. A. Campagnari, Biosens. Bioelectron. 14, 829 (2000).

${ }^{5}$ O. Rotariu, N. J. C. Strachan, and V. Badescu, Phys. Med. Biol. 49, 3971 (2004).

${ }^{6}$ R. Kindervater, W. Künnecke, and R. D. Schmid, Anal. Chim. Acta 234, 113 (1990).

${ }^{7}$ E. Verpoorte, Lab Chip 3, 60N (2003).

${ }^{8}$ J. M. Van Emon and C. L. Gerlach, J. Microbiol. Methods 32, 121 (1998). ${ }^{9}$ Z. Jiang, J. Llandro, T. Mitrelias, and J. A. C. Bland, 50th Annual Conference on Magnetism and Magnetic Materials, San Jose, California USA, 2006 (unpublished).

${ }^{10}$ S. Bronzeau and N. Pamme, Anal. Chim. Acta 609, 105 (2008).

${ }^{11}$ L. G. Rashkovetsky, Y. V. Lyubarskaya, F. Foret, D. E. Hughes, and B. L. Karger, J. Chromatogr., A 781, 197 (1997).

${ }^{12}$ M. A. Hayes, N. A. Polson, A. N. Phayre, and A. A. Garcia, Anal. Chem. 73, 5896 (2001).

${ }^{13}$ K. Nandy, S. Chaudhuri, R. Ganguly, and I. K. Puri, J. Magn. Magn. Mater. 320, 1398 (2008).

${ }^{14}$ T. Baier, S. Mohanty, K. Drese, F. Rampf, J. Kim, and F. Schönfeld, Microfluid. Nanofluid. 7, 205 (2009).

${ }^{15}$ S. Melle, O. G. Calderón, M. A. Rubio, and G. G. Fuller, Phys. Rev. E 68 , 041503 (2003).

${ }^{16}$ E. P. Furlani, J. Appl. Phys. 99, 024912 (2006).

${ }^{17}$ N. Ichikawa, K. Hosokawa, and R. Maeda, J. Colloid Interface Sci. 280, 155 (2004)

${ }^{18}$ N. Modak, A. Datta, and R. Ganguly, Microfluid. Nanofluid. 6, 647 (2009).

${ }^{19}$ S. L. Dance, E. Climent, and M. R. Maxey, Phys. Fluids 16, 828 (2004).

${ }^{20}$ T. R. Strick, J.-F. Allemand, D. Bensimon, A. Bensimon, and V. Croquette, Science 271, 1835 (1996).

${ }^{21}$ A. Sinha, R. Ganguly, and I. K. Puri, J. Magn. Magn. Mater. 321, 2251 (2009). 